\title{
Contingency Management Delivered by Community Therapists in Outpatient Settings
}

Nancy M. Petry

University of Connecticut School of Medicine and Dentistry

Sheila M. Alessi

University of Connecticut School of Medicine and Dentistry

Follow this and additional works at: https://opencommons.uconn.edu/uchcres_articles Part of the Medicine and Health Sciences Commons

\section{Recommended Citation}

Petry, Nancy M. and Alessi, Sheila M., "Contingency Management Delivered by Community Therapists in Outpatient Settings" (2012). UCHC Articles - Research. 158.

https://opencommons.uconn.edu/uchcres_articles/158 


\title{
Contingency management delivered by community therapists in outpatient settings ${ }^{*}$
}

\author{
Nancy M. Petry ${ }^{1,{ }^{*}}$, Sheila M. Alessi ${ }^{1}$, and David M. Ledgerwood ${ }^{2}$ \\ ${ }^{1}$ Calhoun Cardiology Center, University of Connecticut Health Center, Wayne State University \\ School of Medicine \\ ${ }^{2}$ Department of Psychiatry and Behavioral Neurosciences, Wayne State University School of \\ Medicine
}

\section{Abstract}

Background-Few community-based clinicians have been trained to deliver contingency management $(\mathrm{CM})$ treatments, and little data exist regarding the efficacy of $\mathrm{CM}$ when administered by clinicians.

\begin{abstract}
Method-Fifteen clinicians from four intensive outpatient treatment programs received training in CM. Following a didactics seminar and a period in which clinicians delivered CM to pilot patients while receiving weekly supervision, clinicians treated 43 patients randomized to standard care or CM, without supervision. In both treatment conditions, urine and breath samples were collected up to twice weekly for 12 weeks, and CM patients earned the opportunity to win prizes ranging in value from $\$ 1$ to $\$ 100$ for submitting drug-free samples. Primary treatment outcomes were sessions attended, unexcused absences, longest continuous period of abstinence, and proportion of negative samples submitted.
\end{abstract}

Results-All therapists completed the training and supervision phase, and 10 treated randomized patients. Patients randomized to CM achieved significantly greater durations of abstinence than patients randomized to standard care $(5.0 \pm 3.8$ versus $2.6 \pm 3.7$ weeks $)$ and had fewer unexcused absences $(4.3 \pm 1.2$ versus $8.1 \pm 5.4)$, but proportion of negative samples submitted and attendance did not differ significantly between groups. Therapist adherence and competence in CM delivery decreased when supervision was no longer provided, and competence in CM delivery was associated with duration of abstinence achieved and attendance.

Conclusions-Community-based clinicians can effectively administer CM, and outcomes relate to competence in CM delivery. These data call for further training and supervision of community clinicians in this evidence-based treatment.

\section{Keywords}

contingency management; therapist; community clinics; training

\footnotetext{
* Supplementary material can be found by accessing the online version of this paper at http://dx.doi.org and by entering doi:... (C) 2011 Elsevier Ireland Ltd. All rights reserved.

** Corresponding Author: Nancy M. Petry, Calhoun Cardiology Center, University of Connecticut Health Center, 263 Farmington Ave., Farmington, CT, USA, 06030, NPetry@UCHC.edu, Tel. 860-679-2593, Fax. 860-679-1312.

Publisher's Disclaimer: This is a PDF file of an unedited manuscript that has been accepted for publication. As a service to our customers we are providing this early version of the manuscript. The manuscript will undergo copyediting, typesetting, and review of the resulting proof before it is published in its final citable form. Please note that during the production process errors may be discovered which could affect the content, and all legal disclaimers that apply to the journal pertain.
} 


\section{Introduction}

Contingency management (CM) treatments are highly efficacious in improving substance abuse treatment outcomes (Dutra et al., 2008; Lussier et al., 2006; Prendergast et al., 2006). However, CM interventions demonstrated efficacious in research studies are rarely utilized in practice (McGovern et al., 2004), and even in studies in which CM was examined in community-based clinics, therapists working at the sites usually did not administer the treatment. For example, the National Institute on Drug Abuse's Clinical Trials Network (CTN) studies (Peirce et al., 2006; Petry et al., 2005a) examined the efficacy of CM in over 800 stimulant abusing patients from 14 community clinics. Patients were randomized to standard care plus breath and urine testing or that same treatment plus the chance to win prizes contingent upon abstinence. In outpatient drug-free settings (Petry et al., 2005a), the mean (standard deviation; $S D$ ) duration of abstinence increased from 5.2 (6.9) weeks in standard care to 8.6 (9.2) weeks with CM. Although CM was efficacious across community clinics, research assistants, not clinic counselors, administered the CM.

With the publication of these positive results, clinicians have begun using CM in some community-based clinics (Henggeler et al., 2007, 2008; Kellogg et al., 2005; Ledgerwood et al., 2008; Lott and Jencius, 2009; Squires et al., 2008). However, implementation may not be optimal without appropriate understanding of CM principles and sufficient training experiences. Training in an evidence-based treatment involves didactics, observation, and practice, with supervision by individuals experienced in the intervention (Sholomskas et al., 2005). Supervision usually addresses both adherence and competence in therapy delivery. Adherence refers to integrity of the therapy, and competence refers to the quality or skill with which interventions are delivered. In one of the few studies examining adherence to CM principles, Andrzejewski et al. (2001) noted that without regular supervision, clinicians' administration of CM was suboptimal, and clinicians often failed to monitor and reinforce behaviors according to $\mathrm{CM}$ principles. Competence is also important in therapy delivery and has been associated with patient outcomes generally (Barber et al., 1996; Martino et al., 2008). Thus, adherence and competence in CM delivery is needed before widespread implementation, and once clinicians are trained to adhere to CM principles and administer $\mathrm{CM}$ competently, it is necessary to ascertain if patients improve in areas that are clinically important.

This study describes a training program in CM that was administered to clinicians in four outpatient, non-methadone clinics. It also describes outcomes from a randomized trial conducted in these clinics. Inclusion criteria were broad to evaluate effects of CM among patients typically seen in these settings. We expected that once therapists were trained to appropriate standards, CM would enhance durations of abstinence and retention and reduce unexcused absences, a common clinical concern (Dutra et al., 2008; McLellan et al., 2000; Stark, 1992). We also present data on proportion of negative samples submitted, although beneficial effects of $\mathrm{CM}$ on this variable are rarely observed in non-methadone settings because these patients usually submit negative samples so long as they remain engaged in treatment (Petry et al., 2004, 2005a,b, 2006). Results are presented for the sample overall as well as within each clinic to ascertain variability across settings. In addition, we examined associations between competence in CM delivery and patient outcomes, with the expectation that more competent $\mathrm{CM}$ administration would be associated with better outcomes.

\section{Methods}

\subsection{Participants}

Fifteen counselors, employed at four outpatient treatment facilities in 2006 and 2007, participated. The clinics were all not-for-profit and provided intensive outpatient treatment 
as well as continuing aftercare. Inclusion criteria for therapists were: employed at the facility for at least 6 months, at least one year experience working with substance abusing patients, and willingness to allow random assignment of patients. All therapists employed by the clinics at the time of training (two to five per site) participated. Mean (standard deviation; $S D)$ age was $45.6(11.5)$ years, and nine (60.0\%) were women. Three therapists $(20.0 \%)$ were Hispanic, one (6.7\%) was African American, two (13.3\%) were multi-racial, one (6.7\%) was American Indian/Alaskan, and the remainder European American. One (6.7\%) had an associate's degree, three (20.0\%) bachelor's degrees, ten (66.7\%) master's degree, and one (6.7\%) a Psy.D. Four (26.7\%) reported not being in recovery from substance use, and the remainder choose not to answer the question. They had worked with substance abusers for a mean $(S D)$ of 7.7 (6.6) years, and nine (60.0\%) had a license to practice. None had prior exposure to CM.

Patients were 78 ( 35 pilot and 43 randomized) individuals age 18 or older beginning intensive outpatient treatment. The randomized phase had an additional inclusion criterion of abuse or dependence on cocaine, opioids, marijuana, or alcohol. The only exclusionary criteria were significant uncontrolled psychiatric conditions (e.g., suicidality, psychosis) and being in recovery from pathological gambling. This latter criterion was applied because of potential similarity between the reinforcement system and gambling, although no increases in gambling have been reported with prize CM (Petry and Alessi, 2010; Petry et al., 2006).

The baseline assessment consisted of modules adapted from the Structured Clinical Interview for DSM-IV (First et al., 1996) and the Addiction Severity Index (ASI) (McLellan et al., 1985). Figure 1 shows the flow of patients in the study, and patients received $\$ 25$ for completing the baseline assessment. Pilot patients completed abbreviated assessments, containing only information related to inclusion and exclusion criteria.

Therapist and patient participants (including pilot patients, see below) signed informed consent for study participation, including audiotaping of interactions. University and Hospital Institutional Review Boards approved study procedures.

\subsection{Study overview}

The project consisted of three components: a training phase, a supervision phase, and a randomized phase. Each phase is outlined below.

2.2.1. In the training phase-therapists participated in two half-day trainings led by a study author that included didactics, demonstration of alcohol and urine sample testing, and $\mathrm{CM}$ practice with role plays (see below). Before and after the didactics, therapists completed a 20-item general CM knowledge test (Petry and Stitzer, 2002). After the training, they also completed a 20-item multiple choice test about details of this study's CM procedures and a training satisfaction form.

After achieving adequate scores of $\geq 16$ correct on CM knowledge tests, therapists participated in at least three CM role-plays in which they played the part of a therapist and a research assistant assumed the role of a patient. Scenarios included: (1) describing CM to a new patient, (2) providing reinforcement to a patient testing negative, and (3) withholding reinforcement from a patient testing positive. Study authors and research assistants rated audiotaped role plays using the 12-item Contingency Management Competence Scale (CMCS; Petry et al., 2010). Ratings range from 1 ("poor") to 7 ("excellent"), with scores of 4 or greater indicating "acceptable" quality. The CMCS contains two subscales. One relates to Draws and reflects mean ratings on items assessing the discussion of results of sample testing, number of draws earned that session, and number of draws possible at the next session. The second subscale is entitled the General subscale, and it is derived from mean 
ratings on all other items. Interrater reliability, derived from three independent raters, was 0.92. Internal consistency and descriptive, concurrent and predictive validity of the CMCS are good to excellent (Petry et al., 2010). After therapists achieved mean ratings of "4" or higher on all CMCS items on three role plays, they proceeded to a CM supervision phase.

2.2.2 In the supervision phase-therapists delivered $\mathrm{CM}$ as described below to 2-3 pilot patients. A 10-item CM checklist rated adherence and included items about urine and breath sample and draw outcomes as recorded on paper forms (Petry, 2011). Scores ranged from 0 (none) to 10 (perfect) adherence. For example, if a therapist failed to write down results from the toxicology test, the number of draws earned, or the types of prizes won, or the draws earned or prizes won were inconsistent with the reinforcement schedule (see treatment section), a lower score would be given. During weekly supervision, CM experts discussed checklists with therapists.

To evaluate competence in CM, CM sessions were audiotaped and rated via the CMCS. During the supervision phase, research staff provided graphical feedback of CMCS scores weekly. Audiotapes of CM were also reviewed in supervision to point out particularly good, and incorrect, CM administration. After therapists received ratings on the CMCS of "4" or higher for at least three sessions in a row with two pilot patients, they proceeded to the randomized phase.

2.2.3. In the randomized phase-research assistants randomized patients $(N=43)$ to standard care or standard care plus CM using a computerized urn program (Stout et al., 1994) that balanced groups on therapist and baseline toxicology results. Therapists audiorecorded discussions regarding sample testing in both conditions. Research staff monitored therapists' delivery of CM using the CMCS and adherence checklist, but they provided no feedback to therapists in this phase. Blinding individuals to treatments was not possible due to the nature of the intervention.

\subsection{Treatments}

Standard care (SC) was similar across clinics, and included one to four group sessions per day, three to four days a week, during the first four weeks. After four weeks of intensive treatment, care tapered to a minimum of one group session per week. Sessions addressed relapse prevention, coping and life skills training, depression, anxiety, and drug use, AIDS education, and daily planning. In addition to standard care, study patients submitted breath and urine samples twice weekly (e.g., Monday-Thursday, Monday-Friday, Tuesday-Friday) that were screened for alcohol using an Intoximeter (St. Louis, Mo) and for cocaine, opioids and marijuana using onsite Teststicks (Varian, Palo Alto, CA). Sample submission was observed by a same-gender staff member when possible (e.g., study therapist or another therapist if the study therapist was not the same gender as the patient). Samples were collected before or after group therapy sessions, at times mutually convenient to both parties. Therapists were asked to congratulate patients when they tested negative and discuss positive samples as they normally would, encouraging abstinence.

Contingency management consisted of the same treatment as SC, including sample testing. Patients in this condition (in both the supervision and randomized phases) also earned the chance to win prizes for submitting negative samples. Each day they provided a breath sample negative for alcohol $(<.003 \mathrm{~g} / \mathrm{dl})$ and a urine sample negative for cocaine, opioids, and marijuana they earned at least one draw from an urn, each of which provided the chance of winning a prize. Number of draws increased by one for each consecutive set of negative samples submitted, such that the second consecutive day samples tested negative resulted in two draws, the third three draws, and so on up to a maximum of 10 draws per day. Patients 
could earn up to 195 draws if they submitted all negative specimens throughout the 12-week study period.

If patients submitted a sample positive for one of the substances, refused a sample, or had an unexcused absence on a testing day (excused absences included court appearance and medical appointments), they received no draws that day. The next negative sample resulted in one draw. Draws remained low (but still escalated by one for each consecutive negative sample) until three consecutive negative samples were submitted, and then draws returned to the highest level of draws that patient had previously achieved (see also Higgins et al., 2000).

Patients drew from an urn containing 500 cards. Half the cards were not associated with a prize and stated "Good job!" The other half were winning cards: 216 were small $\$ 1$ prizes (e.g., choice of bus tokens, $\$ 1$ fast food coupons, toiletries); 33 were large prizes worth up to $\$ 20$ (e.g., choice of movie theater tickets, CDs, phone cards, watches); and one was a jumbo prize worth up to $\$ 100$ (e.g., choice of stereo or television). Average expected cost per draw was about $\$ 1.95$. Research staff purchased prizes, but clinic therapists completed all other aspects of $\mathrm{CM}$, including screening samples and calculating and administering draws and prizes.

\subsection{Data analysis}

Descriptive data are presented in terms of how therapists met the training criteria, along with their responses to a questionnaire regarding satisfaction with the CM training. Paired $t$-tests compared therapists' scores pre- and post-training on the CM knowledge test.

For patients in the randomized phase, chi-square and $t$-tests compared baseline characteristics between patients randomized to the two conditions. Primary outcomes were: sessions attended, number of unexcused absences on urine testing days, longest duration of abstinence, and proportion of samples that tested negative. Proportion of negative samples was calculated with number of samples submitted in the denominator; the mean $(S D)$ number of samples submitted did not differ by condition, $\mathrm{SC}=10.0$ (5.4) and $\mathrm{CM}=12.7$ (6.6), $t(41)=1.47, p=.15$. A week of abstinence was defined as two consecutively scheduled samples that tested negative for alcohol, cocaine, opioids, and marijuana. If a patient did not attend a scheduled session or refused to provide or missed a sample because of an unexcused absence, the string of abstinence was broken. Consistent with the reinforcement schedule, excused absences (mean <1/patient) did not break an abstinence string if they were preceded and followed by negative samples. Multivariate analysis of variance (MANOVA) evaluated group differences, with the four primary outcomes as dependent variables. Treatment condition and clinic were independent variables (therapist could not be used as an independent variable in these analyses because one therapist treated only one randomized patient, but all clinics had patients randomized to both conditions). Initially, the interaction between treatment condition and clinic was included in the model, but because the interaction was not significant $(p s>.46)$ it was excluded. Results are presented for the sample overall, and within each clinic means and standard deviations are presented. Statistical testing was not conducted within clinics due to the small sample sizes, which were not expected to yield significant difference between groups.

We also compared adherence and competence in CM delivery in the supervision phase to the randomized phase, when no supervision was provided. Univariate ANOVA evaluated differences between the two phases in adherence checklist scores and MANOVA for CMCS subscale scores. In both analyses, therapist and phase (supervision or randomized) were included as independent variables, using only data from therapists who participated in both phases. Finally, we evaluated whether competence in CM delivery during the randomized 
phase was related to patient outcomes. For patients assigned to CM, MANOVA examined predictors of the four outcomes, with therapist and CMCS subscale scores, derived from mean ratings of all sessions for each patient, as independent variables. Analyses were conducted on SPSS (v 15), with $p<.05$ considered significant.

\section{Results}

\subsection{Therapist training}

All 15 therapists completed didactics training. Mean $(S D)$ correct scores on the CM knowledge test pre-training were 12.7 (2.5) of 20, and they rose post-training to $17.7(1.8), t$ $(14)=8.61, p<.001$. On the CM protocol test, therapists answered $18.8(1.2)$ of 20 questions correctly. Mean $(S D)$ scores on role plays were 6.6 (0.6) for the Draw subscale and 6.1 (0.6) for the General subscale, indicating "very good" competence in CM delivery. Fourteen therapists completed training evaluations (Table 1), with all therapists rating the training from "Above Average" to "One of the Best".

In the supervision phase, 15 therapists treated a mean $(S D)$ of $2.5(0.8)$ pilot patients. Demographic characteristics of pilot patients are shown in the left column of Table 2. On average, therapists required 3.8 (2.2) weeks of supervision (range 3-11 weeks) to meet competence criteria, and on average $(S D)$ supervision was provided for a total of 18.2 (12.8) $\mathrm{CM}$ sessions. Scores on the adherence checklist, while high during therapists' first three CM sessions with a mean $(S D)$ of 9.5 (0.9) out of 10 [range 7 to 10 ], rose significantly to 9.9 (0.3) [range 9 to 10] during the last three $\mathrm{CM}$ sessions of the supervision phase, $t(82)=$ $2.79, p=.007$. Scores on the CMCS subscales were also high during the first three sessions of $\mathrm{CM}$ administration and remained high at the end of the supervision period, with respective means $(S D)$ on the Draw subscale of $6.4(1.2)$ and $6.6(0.7)$, and means on the General subscale of $5.8(1.0)$ to $6.1(0.6), p s>.20$. Scores on two items rose significantly. Compared to the initial sessions of CM administration, scores at the end of the supervision phase rose from $5.8(1.2)$ to $6.5(0.7), t(75)=3.41, p<.001$, on the praise effort item, and from $6.2(1.0)$ to $6.6(0.5), t(75)=2.24, p=.03$, on the maintaining session structure item. All 15 therapists completed the supervision phase, but only 10 (66.7\%) proceeded to the randomized phase. Those who discontinued participation cited time difficulties associated with meeting patients for sample collection as the reason.

\subsection{Randomized phase}

Ten therapists treated 43 patients (range 1-13) randomized to SC or CM. Baseline characteristics did not differ between patients assigned to the two conditions (Table 2). In the MANOVA of treatment outcomes, clinic (and the clinic by treatment interaction) was not significant $(p s>.40)$. However, patients randomized to CM had significantly fewer unexcused absences and achieved significantly longer durations of abstinence than those assigned to SC. Means $(S D)$ outcomes for patients overall and in each clinic are presented in Table 3, along with effect sizes comparing CM to SC for the full sample.

\subsubsection{Therapist performance in $\mathrm{CM}$ delivery during the randomized phase-}

Research staff provided therapists with no feedback about CM administration during the randomized phase. On CM adherence checklists, mean $(S D)$ score was 9.2 (1.2) during the randomized phase, which while high, was significantly lower than ratings obtained throughout the supervision phase of $9.6(0.7), F(1,572)=7.75, p=.006$. Similarly, competence in CM administration decreased significantly on the CMCS Draw subscale, $F$ $(6,303)=6.51, p=.01$, and General subscale, $F(6,303)=17.23, p<.001$. Throughout the supervision phase, mean $(S D)$ scores on the Draw and General subscales were $6.7(0.6)$ and $5.9(0.8)$, respectively. In the randomized phase, scores dropped to $6.4(0.9)$ and $5.4(1.0)$ on 
the two respective subscales. Scores on individual items of the CMCS in the supervision and randomized phase are shown in Table 4, and many were significantly lower during the randomized phase. Although therapists' overall average CMSC scores across all CM sessions during the randomized phase remained at an acceptable level, five of $10(50 \%)$ therapists treated one or more CM patients less than adequately in the randomized phase, with scores $<4$.

In examining predictors of patient outcomes, scores on the CMCS Draw subscale were significantly and positively associated with number of sessions attended and longest duration of abstinence achieved, $F(1,11)=7.35, p=.02$ and $F(1,11)=4.89, p=.04$, respectively. Scores on the CMCS General subscale were not related to outcomes, all $p$ s $>$. 37.

3.2.2. Session duration, reinforcement earned, and adverse events-The mean $(S D)$ duration of a CM session, including awarding of prizes, was 6.0 (3.5) minutes. Patients randomized to $\mathrm{CM}$ earned a mean $(S D)$ of 70.5 (63.5) draws, resulting in 32.5 (28.9) small, 4.9 (5.7) large, and $0.2(0.5)$ jumbo prizes, with an overall cost of $\$ 148.0(\$ 155.2)$ per patient. There were no study related adverse events or increases in gambling reported.

\section{Discussion}

This study found that therapists can readily be trained to administer CM with a high level of adherence and competence, and when they deliver CM to patients in outpatient substance abuse treatment facilities, some outcomes are improved relative to standard care. Although positive effects of $\mathrm{CM}$ were noted in the randomized phase, competence in $\mathrm{CM}$ delivery decreased when research staff no longer provided CM supervision, and levels of competence in delivering CM, especially in regards to discussing outcomes of sample testing and draws, were significantly related to patient outcomes. Findings from this study underscore the need for appropriate training and supervision in CM delivery, but they also point to potentially strong beneficial effects associated with using CM in practice.

Regarding the first of these findings, all 15 therapists who participated in the training showed improvements in knowledge about CM, and the training experience was rated favorably on all indices. Few therapists endorsed any conflict between CM principles and their beliefs about effective treatment for substance use. During role plays, therapists readily achieved high levels of competence in CM delivery. Therapists' adherence to the CM protocol in terms of tracking sample results and number of draws was high following the didactics and at the beginning of the supervision phase, and it rose to near perfect levels within an average of 3.8 weeks of individual supervision. Likewise, competence in CM delivery was very good at the beginning of the supervision phase, and increases in competence were observed on two items of the CMCS during the supervision phase. Together, these data demonstrate that this training program was well received and successful, but this study did not compare different training approaches, and other training procedures may also be effective.

With the training approach outlined herein, some beneficial effects of $\mathrm{CM}$ were noted when patients were randomized to $\mathrm{CM}$ versus standard care. Specifically, $\mathrm{CM}$ reduced unexcused absences and enhanced durations of abstinence. However, CM did not affect treatment attendance in this study, and in outpatient clinics, CM usually, although not always (Petry et al., 2004), has effects on increasing retention (Petry et al., 2000, 2006, 2005a). The effect size for improving attendance was in the small to medium range, and it did not reach statistical significance with this sample size. Similarly, effects of CM on reducing proportion of negative samples were not significant, and in the small to medium range. 
Three of the four clinics showed numerical improvements in both these indices, but one clinic did not; in both of these cases, the clinic with the best outcomes for standard care patients showed no numerical improvements with CM. Similarly, for the two variables in which overall effects of $\mathrm{CM}$ were significant, large variations across clinics were observed, with CM seemingly exerting the most benefits when patient outcomes during standard care were relatively poor (i.e., high rates of unexcused absences and low durations of abstinence). Thus, CM may be best suited for outpatient clinics and populations in which outcomes in usual care are poor. Clinicians who implement CM in these settings may observe robust improvements with CM.

Although CM engendered some benefits in the randomized phase, therapists' adherence and competence decreased when they no longer received supervision. Reductions with respect to both monitoring (adherence checklist scores) and delivering CM (CMCS scores) were noted in the randomized relative to supervision phase. Although reductions were minor in magnitude and all therapists retained "adequate" average levels of CM delivery overall, five therapists treated one or more $\mathrm{CM}$ patients at below adequate levels of competence, and several of the differences in CMCS scores between the phases were statistically significant. Because competence in CM delivery as measured on the Draws subscale was related to some patient outcomes, these data suggest that more sustained supervision than that provided in the context of this project may be necessary to ensure high rates of appropriate $\mathrm{CM}$ delivery to optimize patient outcomes.

These results should be interpreted in the context of some limitations. This study was conducted with clinicians working in intensive outpatient clinics, and these results may not generalize to other therapists and settings. These therapists were highly educated, which may have made them more willing to implement CM. As noted previously, we did not evaluate the efficacy of training and supervision methods, and the training, while brief in duration, was individualized. Although therapists continued administering CM adequately after the supervision phase ended, competence and adherence declined significantly on some measures, implying that a longer supervision phase or perhaps booster supervision may be needed to optimize benefits. Psychometric properties of the 10-item adherence checklist have not been formally evaluated, but the CMCS has demonstrated reliability and validity (Petry et al., 2010). Another limitation is that the number of patients in the randomized phase was small, and most therapists treated only a few patients. Finally, one-third of the clinicians choose not to participate in the randomized phase due to time constraints associated with meeting with patients for sample testing, and none of the clinics implemented CM after the study ended. This study provided financial resources and administrative oversight for the prizes, and management of prizes adds further to the administrative costs of CM (Olmstead et al., 2007; Petry, 2011). More work is needed to address issues related to sustainability of CM.

Strengths of this study are that it employed a randomized design in four community-based clinics, and 15 therapists participated. When provided with appropriate CM protocols and training experiences, community-based clinicians were able to implement CM effectively. Importantly, patients who were randomized to the $\mathrm{CM}$ condition showed improvements in clinically important domains. Given the high rates of attrition from treatment and relapse in substance abusing patients (Dutra et al., 2008; McLellan et al., 2000; Stark, 1992), further dissemination of CM and appropriate training and supervision in this evidence-based practice is needed.

\section{Supplementary Material}

Refer to Web version on PubMed Central for supplementary material. 


\section{Acknowledgments}

Funding for this study and preparation of this report was provided by NIH Grants R01-DA016855, P30-DA023918, R01-DA13444, R01-DA018883, R01-DA021567, R01-DA022739, R01-DA024667, R01-DA027615, P50DA09241, P60-AA03510, and M01-RR06192.

\section{References}

Andrzejewski ME, Kirby KC, Morral A, Iguchi MY. Technology transfer through performance management: the effects of graphical feedback and positive reinforcement on drug treatment counselors' behavior. Drug Alcohol Depend. 2001; 63:179-186. [PubMed: 11376922]

Barber JP, Crits-Christoph P, Luborsky L. Effects of therapist adherence and competence on patient outcome in brief dynamic therapy. J. Consult. Clin. Psychol. 1996; 64:619-622. [PubMed: 8698958]

Dutra L, Stathopoulou G, Basden SL, Leyro TM, Powers MB, Otto MW. A meta-analytic review of psychosocial interventions for substance use disorders. Am. J. Psychiatry. 2008; 165:179-187. [PubMed: 18198270]

First, MB.; Spitzer, RL.; Gibbin, M.; Williams, JB. Structured Clinical Interview for DSM-IV Axis I Disorders. Washington, DC: American Psychiatric Press; 1996.

Henggeler SW, Chapman JE, Rowland M, Halliday-Boykins C, Randall J, Shackelford J, Schoenwald SK. If you build it, they will come: statewide practitioner interest in contingency management for youths. J. Subst. Abuse Treat. 2007; 32:121-131. [PubMed: 17306721]

Henggeler SW, Chapman JE, Rowland MD, Halliday-Boykins CA, Randall J, Shackelford J, Schoenwald SK. Statewide adoption and initial implementation of contingency management for substance-abusing adolescents. J. Consult. Clin. Psychol. 2008; 76:556-567. [PubMed: 18665685]

Higgins ST, Wong CJ, Badger GJ, Haug Ogden DE, Dantona RL. Contingent reinforcement increases cocaine abstinence during outpatient treatment and 1 year follow-up. J. Consult. Clin. Psychol. 2000; 68:64-72. [PubMed: 10710841]

Kellogg SH, Burns M, Coleman P, Stitzer M, Wale JB, Kreek MJ. Something of value: the introduction of contingency management interventions into the New York City Health and Hospital Addiction Treatment Service. J. Subst. Abuse Treat. 2005; 28:57-65. [PubMed: 15723733]

Ledgerwood DM, Alessi SM, Hanson T, Godley MD, Petry NM. Contingency management for attendance to group substance abuse treatment administered by clinicians in community clinics. J. Appl. Behav. Anal. 2008; 41:517-526. [PubMed: 19192856]

Lott DC, Jencius S. Effectiveness of very low-cost contingency management in a community adolescent treatment program. Drug Alcohol Depend. 2009; 103:162-165. [PubMed: 19250774]

Lussier JP, Heil S, Mongeon J, Badger G, Higgins ST. A meta-analysis of voucher-based reinforcement therapy for substance use disorders. Addiction. 2006; 101:192-203. [PubMed: $16445548]$

Martino S, Ball S, Nich C, Frankforter TL, Carroll KM. Community program therapist adherence and competence in motivational enhancement therapy. Drug Alcohol Depend. 2008; 96:37-48. [PubMed: 18328638]

McGovern MP, Fox TS, Xie H, Drake RE. A survey of clinical practices and readiness to adopt evidence-based practices: dissemination research in an addiction treatment system. J. Subst. Abuse Treat. 2004; 26:305-312. [PubMed: 15182895]

McLellan AT, Lewis DC, O'Brien CP, Kleber HD. Drug dependence, a chronic medical illness: implications for treatment, insurance, and outcomes evaluation. JAMA. 2000; 284:1689-1695. [PubMed: 11015800]

McLellan AT, Luborsky L, Cacciola J, Griffith J, Evans F, Barr HL, O’Brien CP. New data from the Addiction Severity Index: reliability and validity in three centers. J. Nerv. Ment. Dis. 1985; 173:412-423. [PubMed: 4009158]

Olmstead TA, Sindelar JL, Petry NM. Cost-effectiveness of prize-based incentives for stimulant abusers in outpatient psychosocial treatment programs. Drug Alcohol Depend. 2007; 87:175-182. [PubMed: 16971054] 
Peirce JM, Petry NM, Stitzer ML, Blaine J, Kellogg S, Satterfield F, Schwartz M, Krasnansky J, Pencer E, Silva-Vazquez L, Kirby KC, Royer-Malvestuto C, Roll JM, Cohen A, Copersino ML, Kolodner K, Li R. Effects of lower-cost incentives on stimulant abstinence in methadone maintenance treatment: a National Drug Abuse Treatment Clinical Trials Network study. Arch. Gen. Psychiatry. 2006; 63:201-208. [PubMed: 16461864]

Petry, NM. Contingency Management for Substance Abuse Treatment: A Guide to Implementing this Evidenced-based Practice. New York: Routledge; 2011.

Petry NM, Alessi SM. Prize-based contingency management is efficacious in cocaine-abusing patients with and without recent gambling participation. J. Subst. Abuse Treat. 2010; 39:282-288. [PubMed: 20667679]

Petry NM, Alessi SM, Ledgerwood DM, Sierra S. Psychometric properties of the contingency management competence scale. Drug Alcohol Depend. 2010; 109:167-174. [PubMed: 20149950]

Petry NM, Alessi SM, Marx J, Austin M, Tardiff M. Vouchers versus prizes: contingency management treatment of substance abusers in community settings. J. Consult. Clin. Psychol. 2005b; 73:10051014. [PubMed: 16392974]

Petry NM, Kolodner KB, Peirce JM, Roll JM, Stitzer ML, Hamilton JA. Prize-based contingency management does not increase gambling. Drug Alcohol Depend. 2006; 83:269-273. [PubMed: $16377101]$

Petry NM, Martin B, Cooney JL, Kranzler HR. Give them prizes and they will come: contingency management for treatment of alcohol dependence. J. Consult. Clin. Psychol. 2000; 68:250-257. [PubMed: 10780125]

Petry NM, Peirce JM, Stitzer ML, Blaine J, Roll JM, Cohen A, Obert J, Killeen T, Saladin ME, Cowell M, Kirby KC, Sterling R, Royer-Malvestuto C, Hamilton J, Booth RE, Macdonald M, Liebert M, Rader L, Burns R, DiMaria J, Copersino M, Stabile PQ, Li R. Effects of prize-based incentives on outcomes in stimulant abusers in outpatient psychosocial treatment programs: a National Drug Abuse Treatment Clinical Trials Network study. Arch. Gen. Psychiatry. 2005a; 62:1148-1156. [PubMed: 16203960]

Petry, NM.; Stitzer, ML. Contingency management: using motivational incentives to improve drug abuse treatment. West Haven, CT: Yale University Psychotherapy Development Center; 2002.

Petry NM, Tedford J, Austin M, Nich C, Carroll KM, Rounsaville BJ. Prize reinforcement contingency management for treating cocaine users: how low can we go, and with whom? Addiction. 2004; 99:349-360. [PubMed: 14982548]

Prendergast M, Podus D, Finney J, Greenwell L, Roll J. Contingency management for treatment of substance use disorders: a meta-analysis. Addiction. 2006; 101:1546-1560. [PubMed: 17034434]

Sholomskas DE, Syracuse-Stewert G, Rounsaville BJ, Ball SA, Nuro KF, Carroll KM. We don't train in vain: a dissemination trial of three strategies of training clinicians in cognitive-behavioral therapy. J. Consult. Clin. Psychol. 2005; 73:106-115. [PubMed: 15709837]

Squires DD, Gumbley SJ, Storti S. Training substance abuse treatment organizations to adopt evidence-based practices: the Addiction Technology Transfer Center of New England Science to Service Laboratory. J. Subst. Abuse Treat. 2008; 34:293-301. [PubMed: 17600652]

Stark MJ. Dropping out of substance abuse treatment: a clinically oriented review. Clin. Psychol. Rev. 1992; 12:93-116.

Stout RL, Wirtz PW, Carbonari JP, Del Boca FK. Ensuring balanced distribution of prognostic factors in treatment outcome research. J. Stud. Alcohol Suppl. 1994; 12:70-75. [PubMed: 7723001] 


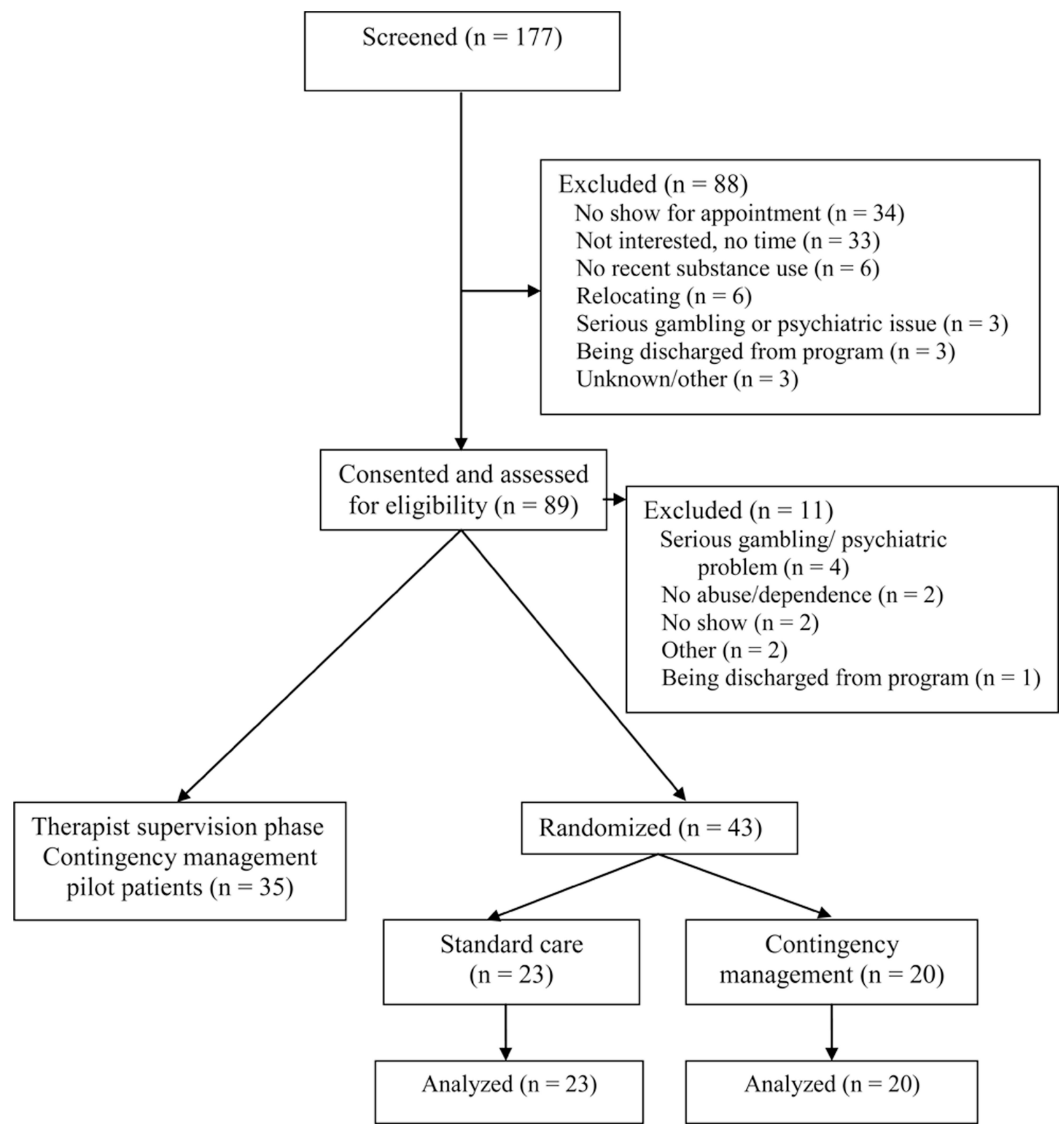

Figure 1.

Flowchart of participants. 
Table 1

Therapists' $(\mathrm{N}=14)$ Ratings on Training Experiences in Contingency Management

\begin{tabular}{lc}
\hline Item & $\begin{array}{c}\text { \% (n) } \\
\text { endorsing }\end{array}$ \\
\hline How does this training rate to others you've received? & \\
Below average & $0.0(0)$ \\
Average & $0.0(0)$ \\
Above average & $28.6(4)$ \\
Very good & $35.7(5)$ \\
One of the best & $35.7(5)$ \\
Would you recommend this training to others? & \\
No, definitely not & $0.0(0)$ \\
No, I don't think so & $0.0(0)$ \\
Yes, I think so & $14.3(2)$ \\
Yes & $7.1(1)$ \\
Yes, enthusiastically & $78.6(11)$ \\
How did this training's materials relate to others? & \\
Much worse & $0.0(0)$ \\
About the same & $0.0(0)$ \\
Somewhat better & $7.1(1)$ \\
Better & $64.3(9)$ \\
Much better & $21.4(3)$ \\
How often do you think contingency management will be helpful with your & \\
clients? & \\
Never & $0.0(0.6(4)$ \\
Rarely & \\
Sometimes & $0.0(0)$ \\
Often & $0.0(0)$ \\
Very often & $0.0(0)$ \\
your belief about what is effective treatment for substance use? & $50.0(7)$ \\
\hline
\end{tabular}




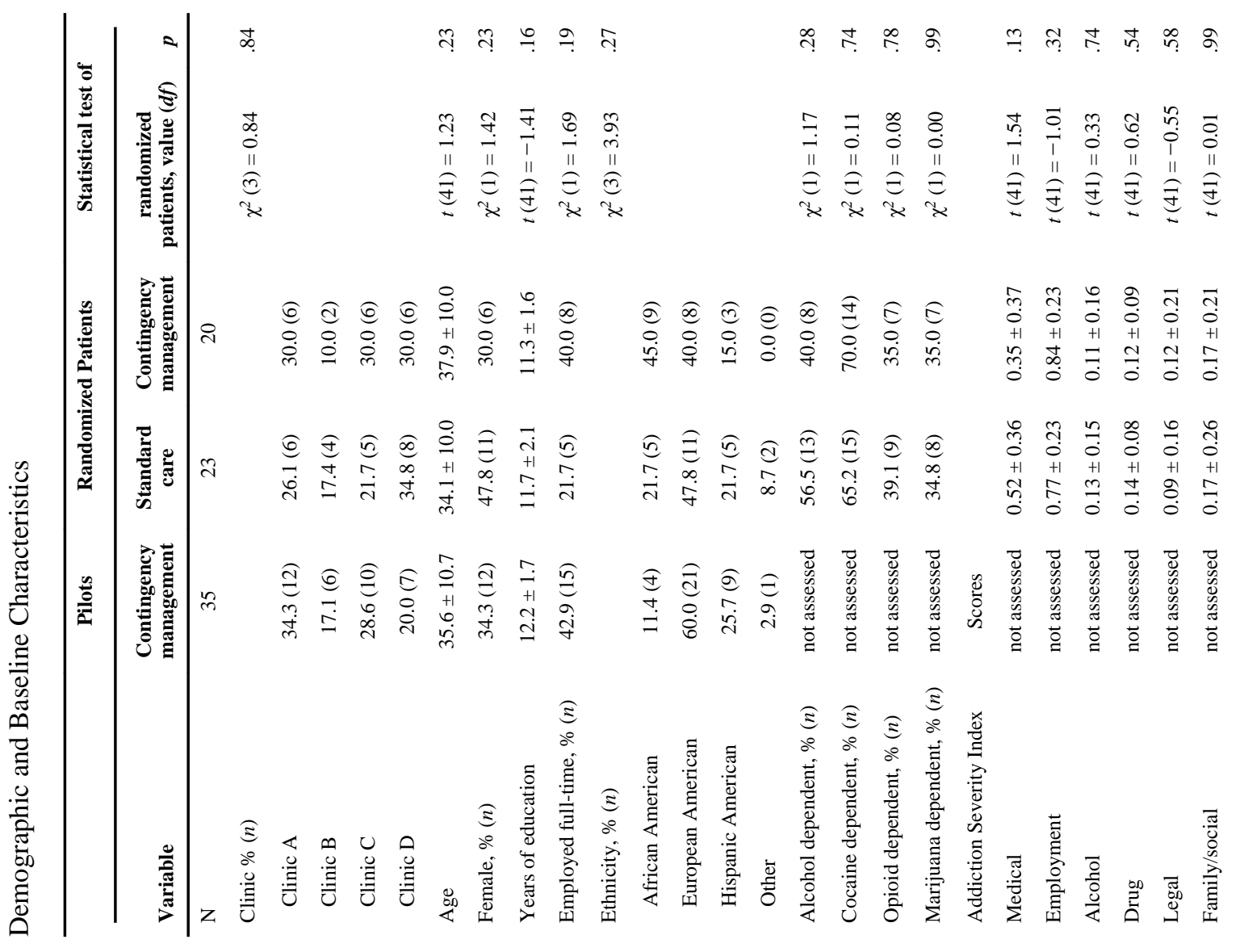




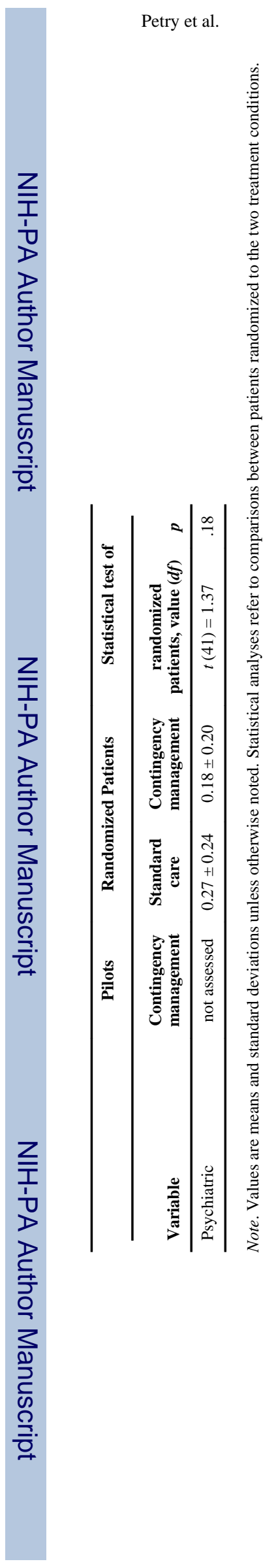

Page 14 
Table 3

Attendance and Abstinence Outcomes

\begin{tabular}{|c|c|c|c|c|}
\hline Variable & $\begin{array}{l}\text { Standard } \\
\text { care }\end{array}$ & $\begin{array}{l}\text { Contingency } \\
\text { management }\end{array}$ & $\begin{array}{c}\text { Statistical test } \\
\text { Value }(d f), p \text {-value }\end{array}$ & $\begin{array}{l}\text { Effect size } \\
\text { Cohen's } d\end{array}$ \\
\hline $\mathbf{N}$ & 23 & 20 & & \\
\hline Sessions attended & $10.3 \pm 6.4$ & $13.0 \pm 6.5$ & $F(1,38)=1.97, p=.17$ & 0.42 \\
\hline Clinic A & $7.8 \pm 4.1$ & $12.0 \pm 6.7$ & & \\
\hline Clinic B & $10.8 \pm 6.4$ & $15.5 \pm 4.9$ & & \\
\hline Clinic C & $9.0 \pm 4.4$ & $14.0 \pm 6.7$ & & \\
\hline Clinic D & $12.5 \pm 7.0$ & $11.3 \pm 8.0$ & & \\
\hline Unexcused absences & $8.1 \pm 5.3$ & $4.3 \pm 1.2$ & $F(1,38)=5.62, p=.02$ & 0.71 \\
\hline Clinic A & $12.0 \pm 8.0$ & $6.0 \pm 5.1$ & & \\
\hline Clinic B & $8.8 \pm 5.3$ & $4.0 \pm 4.2$ & & \\
\hline Clinic C & $9.2 \pm 4.6$ & $3.7 \pm 5.0$ & & \\
\hline Clinic D & $3.3 \pm 3.7$ & $3.1 \pm 3.9$ & & \\
\hline Longest duration of abstinence & $2.6 \pm 3.7$ & $5.0 \pm 3.8$ & $F(1,38)=4.22, p=.04$ & 0.62 \\
\hline Clinic A & $1.8 \pm 1.2$ & $4.3 \pm 3.0$ & & \\
\hline Clinic B & $3.0 \pm 2.4$ & $4.5 \pm 2.1$ & & \\
\hline Clinic C & $0.8 \pm 0.8$ & $5.8 \pm 3.9$ & & \\
\hline Clinic D & $4.5 \pm 4.7$ & $4.8 \pm 5.6$ & & \\
\hline$\%$ Submitted samples negative & $80.5 \pm 28.8$ & $89.6 \pm 29.5$ & $F(1,38)=1.10, p=.30$ & 0.31 \\
\hline Clinic A & $77.8 \pm 40.4$ & $90.9 \pm 20.1$ & & \\
\hline Clinic B & $88.5 \pm 10.4$ & $80.7 \pm 19.8$ & & \\
\hline Clinic C & $69.9 \pm 43.2$ & $92.8 \pm 13.4$ & & \\
\hline Clinic D & $85.3 \pm 25.7$ & $86.7 \pm 32.6$ & & \\
\hline
\end{tabular}

Note. Values represent means and standard deviations. 
Table 4

Mean Ratings on Items from the Contingency Management Competence Scale

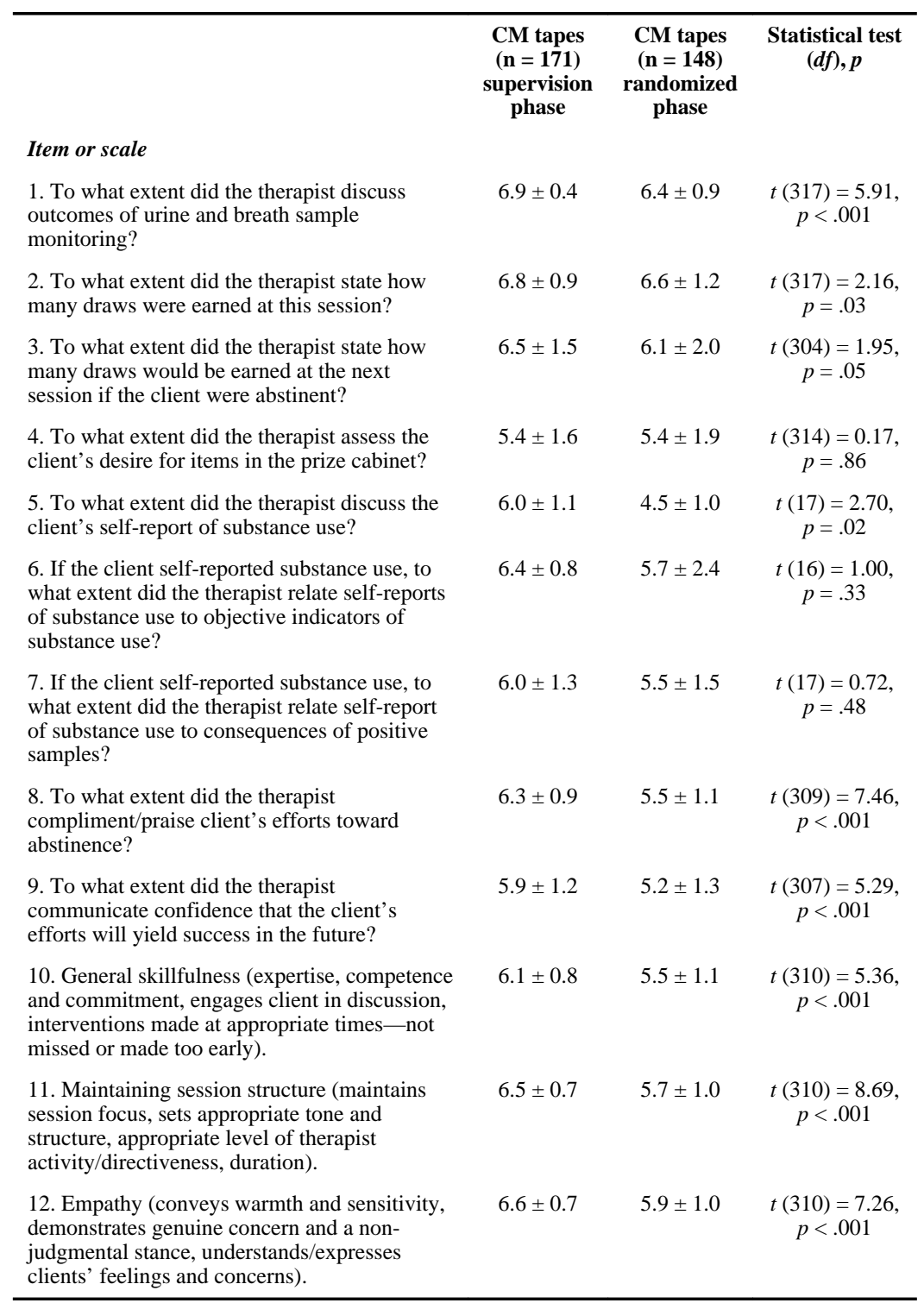

